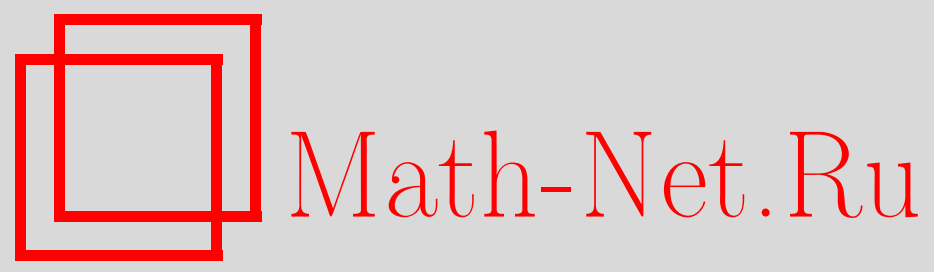

А. С. Абашин, Линейные рекурсивные МДР-коды размерностей 2 и 3, Дискрет. матем., 2000, том 12, выпуск $2,140-153$

DOI: https://doi.org/10.4213/dm325

Использование Общероссийского математического портала Math-Net.Ru подразумевает, что вы прочитали и согласны с пользовательским соглашением http://www .mathnet.ru/rus/agreement

Параметры загрузки:

IP : 52.23 .180 .231

26 апреля 2023 г., 12:56:20

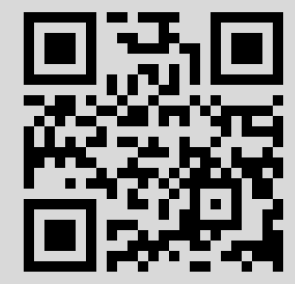


УДК 519.7

\title{
Линейные рекурсивные МДР-коды размерностей 2 и 3
}

\author{
(C) 2000 г. A. C. Абашин
}

\begin{abstract}
Назовем код $\mathscr{K}$ длины $n$ в алфавите $\Omega$ линейным в широком смысле или просто линейным, если существует бинарная операция + на $\Omega$ такая, что $(\Omega,+)$ - абелева группа и $\mathscr{K}$ - подгруппа в $\left(\Omega^{n},+\right)$. Скажем, что $\mathscr{K}$ есть $k$-рекурсивный код, если он состоит из всех слов длины $n \geqslant k$, координаты которых получаются по некоторому фиксированному закону рекурсии из первых $k$ координат.

Пусть $l^{r}(k, q)$ - максимальное $n$, для которого существует линейный $k$ рекурсивный код длины $n$ в алфавите из q элементов с расстоянием $n-k+1$ (МДР-код), a $l^{i r}(k, q)$ - максимальное $n$, для которого существует линейный $k$ рекурсивный идемпотентный (содержащий все слова-константы) МДР-код длины $n$ в алфавите из $q$ элементов. С помощью теории линейных рекуррентных последовательностей найдены значения $l^{i r}(2, q)$ и $l^{r}(3, q)$ для примарного $q$.
\end{abstract}

\section{1. Основные понятия и результаты.}

Пусть $\Omega=\left\{a_{1}, \ldots, a_{q}\right\}-$ произвольное конечное множество. Любое подмножество $\mathscr{K} \subseteq \Omega^{n}$ называется кодом длины $n$ в алфавите $\Omega$. Если $|\mathscr{K}|=q^{k}$, то код называется

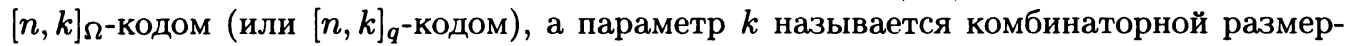
ностью кода $\mathscr{K}$. Расстояние (Хэмминга) $d(u, v)$ между двумя словами $u, v \in \mathscr{K}$ определяется стандартно, как число неравных координат с одинаковыми номерами в $u$ и $v$. Расстояние (Хэмминга) кода $\mathscr{K}$ определяется как минимум $d(\mathscr{K})$ расстояний

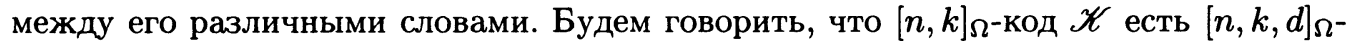
код, если $d(\mathscr{K})=d$. Известно, что $d \leqslant n-k+1$ (граница Синглтона [1]). Код называется МДР-кодом, если $d=n-k+1$. Далее используются результаты и терминология работы [4]. Назовем код $\mathscr{K} \subseteq \Omega^{n}$ линейным в широком смысле, или просто линейным, если существует бинарная операция + на $\Omega$ такая, что $(\Omega,+)-$ абелева группа и $\mathscr{K}$ - подгруппа в $\left(\Omega^{n},+\right)$. В соответствие с классическим определением $[1,2]$ код $\mathscr{K}$ называется линейным, если $\Omega-$ конечное поле и $\mathscr{K}$ является подпространством пространства $\Omega^{n}$ над этим полем. Будем называть такой код линейным в узком смысле. Линейные в широком смысле (над абелевой группой $(\Omega,+)$ ) коды назовем линейно в широком смысле эквивалентными, если один получается из другого перестановкой координат и применением автоморфизмов группы $(\Omega,+)$ к координатам. Назовем $\mathscr{K}$ полным $k$-рекурсивным кодом, если существует функция $f: \Omega^{k} \rightarrow \Omega$, $k \leqslant n$, такая, что $\mathscr{K}$ есть множество всех слов $u(0, n-1)=(u(0), \ldots, u(n-1))$, 
удовлетворяющих условию

$$
u(i+k)=f(u(i), \ldots, u(i+k-1)), \quad i=0,1, \ldots, n-k,
$$

где $u(0), \ldots, u(k-1)$ - произвольные элементы из $\Omega$. Далее такой код обозначается $\mathscr{K}(n, f)$. Будем называть функцию $f: \Omega^{k} \rightarrow \Omega$ и соответствующий код $\mathscr{K}(n, f)$ идемпотентными, если выполняется тождество $f(x, \ldots, x)=x$. Очевидно, что идемпотентность кода $\mathscr{K}$ равносильна тому, что он содержит все слова-константы $\hat{a}=$ $(a, \ldots, a)$, где $a \in \Omega$.

Введем следующие обозначения. Пусть $n(k, q)$ - максимальное $n$ такое, что существует $k$-мерный МДР-код длины $n$ в алфавите мощности $q ; n^{r}(k, q)$ - максимальное $n$ такое, что существует (полный) $k$-рекурсивный МДР-код длины $n$ в алфавите мощности $q ; n^{i r}(k, q)$ - максимальное $n$ такое, что существует (полный) $k$-рекурсивный идемпотентный МДР-код длины $n$ в алфавите мощности $q ; l(k, q)-$ максимум длин линейных $k$-мерных МДР-кодов в алфавите мощности $q$. В случае, когда $q$ - примарное число (степень простого числа), обозначим через $m(k, q)$ такой же параметр в классе кодов линейных в узком смысле.

Аналогично тому, как мы определили $n^{r}(k, q), n^{i r}(k, q)$, можно ввести дополнительно параметры $l^{r}(k, q), l^{i r}(k, q), m^{r}(k, q), m^{i r}(k, q)$ в классах рекурсивных кодов линейных соответственно в широком и в узком смысле. В [4] ставится и частично решается задача оценивания и сравнения элементов матрицы

$$
M(k, q)=\left(\begin{array}{ccc}
{\left[m^{i r}(k, q)\right.} & m^{r}(k, q) & m(k, q)] \\
l^{i r}(k, q) & l^{r}(k, q) & l(k, q) \\
n^{i r}(k, q) & n^{r}(k, q) & n(k, q)
\end{array}\right),
$$

в которой числа, очевидно, не убывают слева направо и сверху вниз. Естественно, первая строка этой матрицы, заключенная в скобки, появляется лишь в случае примарного $q$.

В данной работе сравниваются параметры $l^{r}(k, q)$ и $m^{r}(k, q), l^{i r}(k, q)$ и $m^{i r}(k, q)$ для примарных $q$ и $k=2,3$. Известны следующие результаты:

$$
m^{r}(2, q)=l^{r}(2, q)=n(2, q)=q+1
$$

(см. теорему 6 в [4]);

$$
q-1 \leqslant m^{i r}(2, q) \leqslant l^{i r}(2, q) \leqslant q+1
$$

(см. предложение 10 в [4]); если $p$-простое число, $p \geqslant 3$, то

$$
p \leqslant m^{i r}(2, p) \leqslant l^{i r}(2, p) \leqslant p+1
$$

(см. предложение 11 в [2]); для примарного нечетного $q$

$$
m^{r}(3, q)=l^{r}(3, q)=n^{r}(3, q)=q+1
$$

(см. [1]); если $q=2^{t}$, то

$$
2^{t}+1 \leqslant m^{r}\left(3,2^{t}\right) \leqslant l^{r}\left(3,2^{t}\right) \leqslant m\left(3,2^{t}\right)=2^{t}+2
$$

(см. [1]); и, наконец,

$$
m^{r}(3,4)=5<l^{r}(3,4)=n(3,4)=6, \quad l^{r}(3,8)=9, \quad l^{r}(3,16)=17
$$


(см. [4]).

Равенство $l^{r}(3,4)=6$ было доказано путем построения с помощью ЭВМ линейного в широком смысле $[6,3,4]$-кода над $\mathbf{Z}_{2} \oplus \mathbf{Z}_{2}$, названного Астурийским. Основные результаты данной работы таковы.

Теорема 1. Для примарного q справедливо равенство

$$
l^{\text {ir }}(2, q)= \begin{cases}q, & \text { если } q-\text { простое } \\ q-1 & \text { в противном случае }\end{cases}
$$

Следствие 1. Пусть $F-$ поле из $q=p^{t}$ элементов, $p-$ простое, $t>1$. Тогда любой код $\mathscr{K}$, линейно эквивалентный над группой $(F,+)$ расширенному $[q, 2, q+1]$ коду Рида-Соломона, не является циклическим.

Теорема 2. Для примарного $q \neq 4$ имеет место равенство $l^{r}(3, q)=q+1$.

Следствие 2. Среди кодов, линейно в иироком смысле эквивалентных $\left[2^{t}+2,3,2^{t}\right]$ или $\left[2^{t}+2,2^{t}-1,4\right]$ расширеннъм кодам Рида-Соломона нет циклических кодов.

Таким образом, справедливы следующие уточнения оценок (2), (3), (5). Для любого простого $p$ верны соотношения

$$
p=m^{i r}(2, q)=l^{i r}(2, p)<n(2, p)=p+1
$$

Если $q$ - примарное и не простое число, то

$$
q-1=m^{i r}(2, q)=l^{i r}(2, q)<m^{r}(2, q)=q+1 .
$$

Если $t \geqslant 3$, то

$$
m^{r}\left(3,2^{t}\right)=l^{r}\left(3,2^{t}\right)=2^{t}+1 .
$$

\section{2. Доказательство теоремы 1}

Лемма 1. Пустъ $\mathscr{K}-$ идемпотентный МДР-код размерности 2. Тогда в любом его слове, не являющемся хонстантой, все координаты различны.

Доказательство. Предположим противное. Пусть $\mathscr{K}$ содержит слово $\alpha$ не являющееся константой и такое, что $\alpha(i)=\alpha(j)=a$ для некоторых различных $i, j \in$ $\{1, \ldots, n\}$ и $a \in \Omega$. Тогда $\hat{a} \in \mathscr{K}$, так как $\mathscr{K}-$ идемпотентный код, $\hat{a} \neq \alpha$, так как $\alpha$ - не константа, и $\rho(\hat{a}, \alpha) \leqslant n-2<d(\mathscr{K})=n-1$. Получаем противоречие.

Следствие 3. Длина идемпотентного МДР-кода размерности 2 не превосходит мощности алфавита. Для любого простого $p$ справедливо равенство $l^{\text {ir }}(2, p)=p$.

Доказательство. Действительно, в противном случае в любом слове кода,не являющемся константой есть две одинаковые координаты. Последнее равенство следует теперь из (3). 
Докажем теорему в случае, когда $q=p^{t}$, где $p-$ простое число, $t \in \mathbf{N}, t>1$. Введем некоторые дополнительные понятия. Пусть $\mathscr{K}(n, f)$ - линейный рекурсивный код над абелевой группой $(\Omega,+)$, тогда функция $f: \Omega^{k} \rightarrow \Omega$ есть гомоморфизм группы $\left(\Omega^{k},+\right)$ в группу $(\Omega,+)$ и поэтому она может быть представлена в виде

$$
f\left(x_{1}, \ldots, x_{k}\right)=a_{1}\left(x_{1}\right)+\ldots+a_{k}\left(x_{k}\right),
$$

где $a_{i} \in \operatorname{End}(\Omega,+)$. Для того чтобы код $\mathscr{K}(n, f)$ был МДР-кодом необходимо, чтобы все эндоморфизмы $a_{i}$ были автоморфизмами [4]. Поэтому далее прдполагается, что

$$
a_{i} \in \operatorname{Aut}(\Omega,+), \quad i=1, \ldots, k .
$$

Рассмотрим дополнительно семейство $\mathscr{R}_{\Omega}(f)$ всех рекуррентных последовательностей $u: \mathbf{N}_{0} \rightarrow \Omega$ над $\Omega$, удовлетворяющих условию

$$
u(i+k)=f(u(i, i+k-1)), \quad i \in \mathbf{N}_{0} .
$$

Очевидно, что $\left|\mathscr{R}_{\Omega}(f)\right|=q^{k}$ и

$$
\mathscr{K}(n, f)=\mathscr{R}_{\Omega}^{0, n-1}(f)
$$

есть множество всех начальных отрезков $u(0, n-1)=(u(0), \ldots, u(n-1))$ длины $n$ рекуррент $u \in \mathscr{R}_{\Omega}(f)$. Ввиду конечности $\Omega$ и условия (10) все последовательности из $\mathscr{R}_{\Omega}(f)$ являются реверсивными (чисто периодическими). Наименьшее общее кратное периодов $T(u)$ последовательностей $u \in \mathscr{R}_{\Omega}(f)$ назовем периодом функции $f$ и обозначим $T(f)$.

Ввиду определений и соотношений (10) достаточно доказать, что не существует идемпотентного линейного рекурсивного $[q, 2, q-1]$-кода над абелевой группой $(\Omega,+)$ порядка $q$. Пусть $\mathscr{K}=K\left(q, f\left(x_{1}, x_{2}\right)\right)$ - такой код.

Покажем прежде всего, что тогда $\Omega$ - элементарная абелева группа. Справедливо разложение

$$
\Omega \cong \mathbf{Z}_{p^{i_{1}}} \oplus \ldots \oplus \mathbf{Z}_{p^{i_{s}}}
$$

Без ограничения общности можно считать, что $i_{1} \geqslant \ldots \geqslant i_{s} \geqslant 1$. Предположим, что $i_{1}>1$. Рассмотрим произвольное слово $\alpha=(\alpha(1), \ldots, \alpha(q)) \in \mathscr{K}$, не являющееся константой. По лемме 1 в слове $\alpha$ нет одинаковых координат. Значит, множество $\{\alpha(1), \ldots, \alpha(q)\}$ сопадает с $\Omega$ и в этом множестве есть по крайней мере $p$ элементов порядка $p$. Ввиду линейности кода $\mathscr{K}$ заключаем, что $p^{i_{1}-1} \alpha$ принадлежит $\mathscr{K}$ и $p^{i_{1}-1} \alpha-$ ненулевое слово, содержащее по крайней мере $p$ нулей. Получаем противоречие. Таким образом, доказано, что $\Omega \cong \mathbf{Z}_{p} \oplus \ldots \oplus \mathbf{Z}_{p}$. Будем считать, что

$$
\Omega=\left(\mathbf{Z}_{p}\right)^{(t)}
$$

есть пространство столбцов длины $t$ над $\mathbf{Z}_{p}$.

Покажем, что $\mathscr{K}-$ циклический код. Пусть $\alpha \in \mathscr{K}$ - произвольное слово, отличное от константы. Ввиду рекурсивности кода $\mathscr{K}$ ему принадлежит также слово вида $(\alpha(2), \ldots, \alpha(q-1), \beta)$, которое тоже не является константой (см. лемму 1$)$. Следовательно,

$$
\{\alpha(2), \ldots, \alpha(q), \beta\}=\Omega=\{\alpha(1), \ldots, \alpha(q)\}
$$

то есть $\beta=\alpha(1)$. 
Из свойства цикличности кода $\mathscr{K}$ и леммы 1 следует, что все последовательности $u \in \mathscr{R}_{\Omega}(f)$, не являющиеся константами, имеют период $T(u)=q$, и значит, $T(f)=q$.

Рассмотрим отображение $\pi_{i}: \Omega \rightarrow \mathbf{Z}_{p}$, ставящее в соответствие произвольному элементу $\omega=\left(\omega_{1} \ldots \omega_{t}\right)^{T}$ его $i$-ю координату: $\pi_{i}(\omega)=\omega_{i}$. Для каждой последовательности $u \in \mathscr{R}_{\Omega}(f)$ обозначим через $\pi_{i}(u)$ последовательность над $\mathbf{Z}_{p}$, состоящую из $i$-х координат членов последовательности $u$ :

$$
\pi_{i}(u)=\left(\pi_{i}(u(0)), \pi_{i}(u(1)), \ldots\right),
$$

и обозначим через $L_{i}=\pi_{i}\left(\mathscr{R}_{\Omega}(f)\right)$ множество всех таких различных последовательностей. Очевидно, что это множество замкнуто относительно операций сдвига и сложения последовательностей. Поэтому (см., например $[4,5]) \pi_{i}\left(\mathscr{R}_{\Omega}(f)\right)$ есть ЛРПсемейство над $\mathbf{Z}_{p}$, то есть существует унитарный многочлен $g_{i}(x) \in \mathbf{Z}_{p}[x]$ такой, что $L_{i}=L_{\mathbf{Z}_{p}}\left(g_{i}(x)\right)$ есть множество всех линейных рекуррентных последовательностей (ЛРП) с характеристическим многочленом $g_{i}(x)$. Наименьшее общее кратное периодов последовательностей из $L_{\mathbf{Z}_{p}}\left(g_{i}(x)\right)$ равно периоду $T\left(g_{i}(x)\right)$ многочлена $g_{i}(x)$, поэтому

$$
\left[T\left(g_{1}(x)\right), \ldots, T\left(g_{t}(x)\right)\right]=T(f)=q .
$$

Следовательно, для каждого $i=1, \ldots, t$ выполняется соотношение $T\left(g_{i}(x)\right)=p^{s_{i}}$, причем $1 \leqslant s_{i} \leqslant t$ и одно из чисел $s_{1}, \ldots, s_{t}$ равно $t$.

Пусть $s_{1}=t$. В таком случае (см., например $\left.[3,8]\right)$

$$
g_{1}(x)=(x-e)^{r}, \quad p^{t-1}<r \leqslant p^{t},
$$

и справедливо равенство

$$
\left|L_{1}\right|=p^{r}
$$

Пусть $K_{1}=L_{1}^{0, q-1}-$ множество всех начальных отрезков длины $q$ линейных рекуррент из $L_{1}$. Согласно (15) верно соотношение

$$
\left|K_{1}\right|=p^{r} .
$$

Отображение $\pi_{1}^{n}: \mathscr{K} \rightarrow K_{1}$, ставящеее в соответствие каждому слову $\alpha \in \mathscr{K}$ слово

$$
\pi_{1}^{n}(\alpha)=\left(\pi_{1}(\alpha(1)), \ldots, \pi_{1}(\alpha(q))\right) \in K_{1}
$$

есть, очевидно, эпиморфизм групп и потому

$$
\left|\operatorname{Ker} \pi_{1}^{n}\right|=\frac{|\mathscr{K}|}{\left|K_{1}\right|}=p^{2 t-r} .
$$

С другой стороны, $\operatorname{Ker} \pi_{1}^{n}$ состоит из всех таких слов $\alpha \in \mathscr{K}$, у которых координаты удовлетворяют условию $\pi_{1}(\alpha(t))=0, t=1, \ldots, q$. Так как общее число элементов $a \in$ $\Omega$ со свойством $\pi_{1}(a)=0$ есть $p^{t-1}<q$, в каждом слове $\alpha \in \operatorname{Ker} \pi_{1}^{n}$ есть одинаковые координаты и ввиду леммы $1 \alpha$ - константа. Отсюда следует, что $\left|\operatorname{Ker} \pi_{1}^{n}\right|=p^{t-1}$ и ввиду (14), (16), (17) выполняются соотношения $r=t+1>p^{t-1}$. Последнее неравенство возможно только если $p^{t}=4$, но в этом случае равенство $l^{\text {ir }}(2, q)=q-1$ отмечено в [3] и легко проверяется вручную.

Для доказательства следствия 1 достаточно заметить, что если код является циклическим, то это - линейный рекурсивный код размерности 2 над группой $(F,+)$. 


\section{3. Доказательство теоремы 2}

Ввиду (4), (6) остается рассмотреть лишь случай, когда $q=2^{t}, t>4$, что и предполагается далее. Ввиду (5) достаточно доказать, что не существует линейного рекурсивного $[q+2,3, q]$-кода.

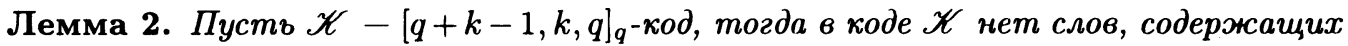
ровно $k-2$ нулей, то есть нет слов веса $q+1$.

Доказательство. Пусть $A_{n-i}$ - число слов линейного $[n, k, n-k+1]$-кода, имеющих ровно $i$ нулевых координат. В [2] выведена формула

$$
A_{n-i}=\sum_{m=0}^{k-1}(-1)^{m-i}\left(\begin{array}{c}
m \\
i
\end{array}\right)\left(\begin{array}{c}
n \\
m
\end{array}\right)\left(q^{k-m}-1\right) .
$$

Отсюда при условии $n=q+k-1$ следует равенство $A_{n-(k-2)}=0$.

Для натуральных чисел $a_{1}, \ldots, a_{n}$ будем обозначать $\left(a_{1}, \ldots, a_{n}\right)$ их наибольший общий делитель, напомним, что $\left[a_{1}, \ldots, a_{n}\right]$ обозначает наименьшее общее кратное этих чисел.

Лемма 3. Для любых натуралъных $n$ u

$$
\left(2^{n}-1,2^{m}+1\right)=\frac{2^{(n, 2 m)}-1}{2^{(n, m)}-1}
$$

$u 2^{m}+1$ делит $2^{n}-1$ тогда и только тогда, когда $2 m$ делит $n$.

Доказателъство. Известно, что для произвольных натуральных $a$ и $b$ справедливо равенство

$$
\left(2^{a}-1,2^{b}-1\right)=2^{(a, b)}-1 .
$$

При $a=n, b=2 m$

$$
\left(2^{n}-1,2^{2 m}-1\right)=2^{(n, 2 m)}-1 .
$$

Так как $\left(2^{m}-1,2^{m}+1\right)=1$, получаем цепочку равенств

$$
\left(2^{n}-1,2^{2 m}-1\right)=\left(2^{n}-1,2^{m}+1\right)\left(2^{n}-1,2^{m}-1\right)=\left(2^{n}-1,2^{m}+1\right)\left(2^{(n, m)}-1\right) .
$$

Отсюда следует (19).

Докажем второе утверждение. Если $2^{m}+1$ делит $2^{n}-1$, то $\left(2^{n}-1,2^{m}+1\right)=2^{m}+1$ и справедливо равенство $(n, 2 m)=2(n, m)$, так как иначе $(n, 2 m)=(n, m)$ и правая часть равенства (19) равна 1 . Таким образом $n$ делится на $2(n, m)$ и ввиду (19)

$$
2^{m}+1=\left(2^{n}-1,2^{m}+1\right)=2^{(n, m)}+1 .
$$

Следовательно, $(n, m)=m$ и $2 m$ делит $n$.

Обратно. Пусть $2 m$ делит $n$. Тогда ввиду (19)

$$
\left(2^{n}-1,2^{m}+1\right)=\frac{2^{2 m}-1}{2^{m}-1}=2^{m}+1
$$

то есть $2^{m}+1$ делит $2^{n}-1$. 
Следствие 4. Условие $\left(2^{n}-1,2^{m}+1\right) \neq 1$ выполняется тогда и только тогда, когда $n /(n, m)$ - четное число, $m /(n, m)$ - нечетное число. Если выполняются два последних условия, то $\left(2^{n}-1,2^{m}+1\right)=2^{(n, m)}+1$.

Доказательство. Как уже отмечалось при доказательстве леммы 3, условие $\left(2^{n}-1,2^{m}+1\right) \neq 1$ выполняется тогда и только тогда, когда $(n, 2 m)=2(n, m)$. В этом случае $(n /(n, m), 2 m /(n, m))=2$ и так как $n /(n, m)$ и $m /(n, m)$ - взаимно простые числа,

$$
(n /(n, m), 2 m /(n, m))=(n /(n, m), 2)=2,
$$

то есть 2 делит $n /(n, m)$. Нечетность же числа $m /(n, m)$ следует из его взаимной простоты с $n /(n, m)$.

Следующий известный результат можно легко доказать методом математической индукции по параметру $n$.

Лемма 4. Пусть $a_{1}, \ldots, a_{n}$ - произво́лънъе натуральные числа. Тогда справедлива формула

$$
\left[a_{1}, \ldots, a_{n}\right]=a_{1} \ldots a_{n} \prod_{r=2}^{n} \prod_{1 \leqslant i_{1}<\ldots<i_{r} \leqslant n}\left(a_{i_{1}}, \ldots, a_{i_{r}}\right)^{(-1)^{r-1}} .
$$

Лемма 5. Пусть $t$ - целое число, большее 1 и не равное степени числа 2 , и пусть $p_{1}, \ldots, p_{n}$ - различные простые нечетные делители числа $t$. Тогда верно неравенство

$$
\left[2^{t / p_{1}}+1, \ldots, 2^{t / p_{n}}+1\right]<2^{t}+1 \text {. }
$$

Доказательство. Рассмотрим число

$$
C=\ln \frac{\left[2^{t / p_{1}}+1, \ldots, 2^{t / p_{n}}+1\right]}{2^{t}+1} .
$$

Чтобы доказать лемму, достаточно показать, что $C<0$. Положим $a_{i}=2^{t / p_{i}}+1$, $i=1, \ldots, n$. С помощью леммы 3 вычислим наименьшее общее кратное $\left[a_{1}, \ldots, a_{n}\right]$. Для этого найдем сначала наиболыние общие делители $\left(a_{i_{1}}, \ldots, a_{i_{r}}\right)$, где $1 \leqslant i_{1}<$ $\ldots<i_{r} \leqslant n$. Покажем, что $\left(a_{i_{1}}, \ldots, a_{i_{r}}\right)=\delta$, где $\delta=2^{t /\left(p_{i_{1}} \ldots p_{i_{r}}\right)}+1$. Заметим, что $\delta$ делит $a_{i_{s}}$ для любого $s=1, \ldots, r$, так как все $p_{1}, \ldots, p_{n}$ - нечетные числа. Значит, $\delta$ делит $\left(a_{i_{1}}, \ldots, a_{i_{r}}\right)$. С другой стороны, из леммы 3 следует, что $\left(a_{i_{1}}, \ldots, a_{i_{r}}\right)$ есть число вида $2^{d}+1$ для некоторого $d \in \mathbf{N}$, которое делит $t / p_{i_{s}}$ для каждого $s=1, \ldots, r$, то есть делит $\left(t / p_{i_{1}}, \ldots, t / p_{i_{r}}\right)$. Так как $p_{1}, \ldots, p_{n}$ - различные простые числа, $\left(t / p_{i_{1}}, \ldots, t / p_{i_{r}}\right)=t /\left(p_{i_{1}} \ldots p_{i_{r}}\right)$. Таким образом, $d$ делит $t /\left(p_{i_{1}} \ldots p_{i_{r}}\right)$ и $\left(a_{i_{1}}, \ldots, a_{i_{r}}\right)$ не больше $\delta$, то есть $\delta=\left(a_{i_{1}}, \ldots, a_{i_{r}}\right)$.

Теперь, применяя формулу (20) для $\left[a_{1}, \ldots, a_{n}\right]$, находим, что

$$
C=\sum_{r=0}^{n} \sum_{1 \leqslant i_{1}<\ldots<i_{r} \leqslant n}(-1)^{r-1} \ln \left(1+2^{t /\left(p_{i_{1}} \ldots p_{i_{r}}\right)}\right) .
$$

Рассмотрим число

$$
D=\sum_{r=0}^{n} \sum_{1 \leqslant i_{1}<\ldots<i_{r} \leqslant n}(-1)^{r-1} \ln 2^{t /\left(p_{i_{1}} \ldots p_{i_{r}}\right)}
$$


Используя несложные преобразования, получаем цепочку соотношений

$$
\begin{aligned}
D & =\sum_{r=0}^{n} \sum_{1 \leqslant i_{1}<\ldots<i_{r} \leqslant n} \frac{(-1)^{r-1} t}{p_{i_{1}} \ldots p_{i_{r}}} \ln 2 \\
& =-\frac{t}{p_{1} \ldots p_{n}} \ln 2 \sum_{r=0}^{n} \sum_{1 \leqslant i_{1}<\ldots<i_{r} \leqslant n}(-1)^{n-r} p_{i_{1}} \ldots p_{i_{r}} \\
& =-\frac{t}{p_{1} \ldots p_{n}}\left(p_{1}-1\right) \ldots\left(p_{n}-1\right) \ln 2<-2 .
\end{aligned}
$$

Представляя $C$ в виде (21), а $D$ в виде (22), получим оценку

$$
\begin{aligned}
C-D & =\sum_{r=0}^{n} \sum_{1 \leqslant i_{1}<\ldots<i_{r} \leqslant n}(-1)^{r-1} \ln \left(1+2^{-t /\left(p_{i_{1}} \ldots p_{i_{r}}\right)}\right) \\
& <\sum_{r=0}^{n} \sum_{1 \leqslant i_{1}<\ldots<i_{r} \leqslant n} \ln \left(1+2^{-t /\left(p_{i_{1}} \ldots p_{i_{r}}\right)}\right) .
\end{aligned}
$$

Отсюда, пользуясь тем, что для любого положительного $x \in \mathbf{R}$ справедливо неравенство $\ln (1+x)<x$, получаем, что

$$
C-D<\sum_{r=0}^{n} \sum_{1 \leqslant i_{1}<\ldots<i_{r} \leqslant n} 2^{-t /\left(p_{i_{1}} \ldots p_{i_{r}}\right)} \leqslant \sum_{r=0}^{t} 2^{-r}<2<-D .
$$

Таким образом $C<0$.

Предположим, что утверждение теоремы неверно. Тогда существует линейный рекурсивный $[q+2,3, q]_{q}$-код $\mathscr{K}$. Как уже отмечалось ранее, в этом случае $q=2^{t}$. Так же, как и при доказательстве теоремы 1 , доказывается,что $\Omega \cong \mathbf{Z}_{2} \oplus \ldots \oplus \mathbf{Z}_{2}$. Будем в дальнейшем считать, что $\Omega=\left(\mathbf{Z}_{2}\right)^{(t)}-$ пространство столбцов длины $t$ над $\mathbf{Z}_{2}$. Тогда закон $f\left(x_{1}, x_{2}, x_{3}\right)$ рекурсии кода можно записать в виде

$$
f\left(x_{1}, x_{2}, x_{3}\right)=A_{0} x_{1}+A_{1} x_{2}+A_{2} x_{3},
$$

где $A_{i}$ - обратимая $t \times t$ матрица над $\mathbf{Z}_{2}$ для $i=0,1,2$.

Для любой последовательности $u$ над $\Omega$ положим

$$
u(i)^{\downarrow}=(u(i), u(i+1), u(i+2))^{T} .
$$

Нетрудно заметить, что в таком случае любая последовательность $u \in \mathscr{R}_{\Omega}(f)$ удовлетворяет равенствам

$$
u(i)^{\downarrow}=S(f)^{i} u(0)^{\downarrow}, \quad i \in \mathbf{N}_{0},
$$

где $S(f)$ - сопровождающая матрица закона рекурсии

$$
S(f)=\left(\begin{array}{ccc}
0 & E & 0 \\
0 & 0 & E \\
A_{0} & A_{1} & A_{2}
\end{array}\right)
$$


(здесь $E=E_{t \times t}-$ единичная $t \times t$ матрица).

Пусть $m_{f}(x) \in \mathbf{Z}_{2}[x]-$ минимальный многочлен матрицы $S(f)$. Тогда

$$
\operatorname{deg}\left(m_{f}(x)\right) \leqslant \operatorname{dim}_{\mathbf{Z}_{2}} \Omega^{3}=3 t
$$

и несложно доказать, что мультипликативный порядок $S(f)$ в группе всех обратимых матриц размера $3 t \times 3 t$ совпадает с $T(f)$ и равен периоду $T\left(m_{f}(x)\right)$ многочлена $m_{f}(x)$.

Лемма 6. Для любой последователъности $u \in \mathscr{R}_{\Omega}(f)$ из условия $u(0)=0$ следует, что $u(q+2)=0$.

Доказательство. Пусть $u \in \mathscr{R}_{\Omega}(f)$ и $u(0)=0$. Слово $(u(0), \ldots, u(q+1))$ лежит в $\mathscr{K}$ и содержит по крайней мере один нуль. По лемме 2 код $\mathscr{K}$ не содержит ненулевых слов с одной нулевой координатой. Значит, существует $i \in\{1, \ldots, q+1\}$ такое, что $u(i)=0$. Единственность такого $i$ следует из того, что $\mathscr{K}-$ МДР-код и потому он не содержит ненулевого слова веса меньшего $q$. Ввиду $(11)$ слово $(u(1), \ldots, u(q+2))$ также лежит в $\mathscr{K}$, но среди элементов $u(1), \ldots, u(q+1)$ ровно один равен нулю. Значит, по лемме $2 u(q+2)=0$.

Лемма 7. Справедливо равенство

$$
S(f)^{q+2}=\left(\begin{array}{ccc}
H & 0 & 0 \\
0 & H & 0 \\
0 & 0 & H
\end{array}\right)
$$

где $H$ - обратимая $t \times t$ матрица, перестановочная с матричами $A_{0}, A_{1}, A_{2}$.

Дохазательство. Из (23) находим, что

$$
u(q+2)^{\downarrow}=S(f)^{q+2} u(0)^{\downarrow}, \quad u(0)^{\downarrow} \in \Omega^{(3)} .
$$

Следовательно, существует $t \times t$ матрицы $B_{1}, B_{2}, B_{3}$ над $\mathbf{Z}_{2}$ такие, что

$$
u(q+2)=B_{1} u(0)+B_{2} u(1)+B_{3} u(2) .
$$

Очевидно, что если среди матриц $B_{2}$ и $B_{3}$ есть ненулевая, то при условии $u(0)=0$ всегда можно подобрать значения $u(1)$ и $u(2)$, при которых $u(q+2) \neq 0$. Но согласно лемме 6 это невозможно. Значит, $B_{2}=B_{3}=0$ и матрица $H=B_{1}$ удовлетворяет условию $u(q+2)=H u(0)$. Так как $\mathscr{K}-$ рекурсивный код, очевидно, что $u(q+2+i)=H u(i)$ для каждого $i \in \mathbf{N}_{0}$. Отсюда следует, в частности, что матрица $S(f)^{q+2}$ имеет указанный вид. Обратимость матрицы $H$ следует из обратимости $S(f)$. Матрица $S(f)^{q+2}$ перестановочна с $S(f)$, поэтому $H$ перестановочна с $A_{i}$ для каждого $i=0,1,2$.

Лемма 8. Для любого $i \geqslant q+2 u$ для любой последовательности $u \in \mathscr{R}_{\Omega}(f)$ справедливъ импликации

$$
u(i)=0 \Longleftrightarrow u(i-(q+2))=0 .
$$

Доказателъство. Утверждение следует из леммы 7 и равенства

$$
u(i)^{\downarrow}=S(f)^{q+2} u(i-(q+2))^{\downarrow},
$$

вытекающего из (23). 
Лемма 9. Матрица $H$ из леммы 7 имеет неприводимый минимальный многочлен наd $\mathrm{Z}_{2}$.

Дожазательство. Если минимальный многочлен матрицы $H$ приводим, то он имеет собственный делитель $g(x) \in \mathbf{Z}_{2}[x]$. В таком случае $\operatorname{Ker} g(H)=\Omega_{1}$ - собственное подпространство пространства $\Omega$ мощности $q_{1}<q$. Из перестановочности $H$ с $A_{0}$, $A_{1}, A_{2}$ следует, что если последовательность $u \in \mathscr{R}_{\Omega}(f)$ удовлетворяет условию

$$
u(0), u(1), u(2) \in \Omega_{1},
$$

то все ее члены также принадлежат $\Omega_{1}$. Таким образом подмножество $\mathscr{K}_{1} \subset \mathscr{K}$ вида

$$
\mathscr{K}_{1}=\left\{\alpha \in \mathscr{K}: \alpha(0), \alpha(1), \alpha(2) \in \Omega_{1}\right\}
$$

есть линейный рекурсивный $[q+2,3, q]$-код в алфавите $\Omega_{1}$. Такого кода не существует, так как согласно (5)

$$
l^{r}\left(3, q_{1}\right) \leqslant q_{1}+2<q+2 .
$$

Следовательно минимальный многочлен матрицы $H$ неприводим.

Следствие 5. Пустъ $T(H)$ - мулътипликативный порядок матричы $H$ в группе обратимых $t \times t$ матриц. Тогда имеет место равенство

$$
T(f)=(q+2) T(H)
$$

и $T(H)$ - нечетное число.

Доказательство. Из леммы 7 следует, что $S(f)^{(q+2) T(H)}=E$ (здесь $E=E_{3 t \times 3 t}-$ единичная $3 t \times 3 t$ матрица), и так как $T(f)=T(S(f))$, то $T(f)$ делит $T(H)(q+2)$. Для доказательства (24) теперь достаточно показать, что $T(H)(q+2)$ делит $T(f)$. Рассмотрим произвольную ненулевую последовательность $u \in \mathscr{R}_{\Omega}(f)$, удовлетворяющую условию $u(0)=u(1)=0$. Тогда $u(i(q+2))=u(i(q+2)+1)=0$ для каждого $i \in \mathbf{N}_{0}$ и других нулей в последовательности нет. Действительно, если $u(s)=0$ для некоторого $s \in \mathbf{N}_{0}$, которое не сравнимо с 0 или 1 по модулю $q+2$, то для подходящего $i \in \mathbf{N}_{0}$ выполняются неравенства

$$
1+i(q+2)<s<(i+1)(q+2)
$$

и коду $\mathscr{K}$ принадлежит ненулевое слово

$$
(u(i(q+2)), u(i(q+2)+1), \ldots, u((i+1)(q+2)-1)),
$$

содержащее 3 нуля, что невозможно. Отсюда следует, что $(q+2)$ делит $T(u)$, а значит, $(q+2)$ делит $T(f)$. Пусть $T(f)=(q+2) m_{1}$. Для произвольной ненулевой последовательности $u \in \mathscr{R}_{\Omega}(f)$ верны соотношения $u(T(f))=u(0)$, так как $T(f)-$ период $\mathscr{R}_{\Omega}(f)$, и

$$
u(T(f))=u\left((q+2) m_{1}\right)=H^{m_{1}} u(0) .
$$

Значит, $m_{1}$ делит $T(H)$ и потому $T(H)(q+2)$ делит $T(f)$. Следовательно, верно (24). Так как минимальный многочлен матрицы $H$ неприводим, из [3] следует, что $T(H)$ - нечетное число. 
Лемма 10. Пусть $m_{f}(x)=g_{1}(x)^{d_{1}} \ldots g_{l}(x)^{d_{l}}$ - каноническое разложение многочлена $m_{f}(x)$, в котором неприводимые многочлены имеют степени $n_{1}, \ldots, n_{l}$ соответственно. Тогда $d_{i} \leqslant 2, i=1, \ldots, l$, и один из многочленов $g_{1}(x), \ldots, g_{l}(x)$ имеет степень $2(t-1)$.

Доказателъство. По следствию 5

$$
T\left(m_{f}(x)\right)=T(f)=(q+2) T(H)=2\left(2^{t-1}+1\right) T(H)
$$

и $\left(2^{t-1}+1\right) T(H)-$ нечетное число. Следовательно (см., например, [3]), имеют место соотношения

$$
\max \left\{d_{1}, \ldots, d_{l}\right\}=2, \quad T\left(g_{1}(x) \ldots g_{l}(x)\right)=\left(2^{t-1}+1\right) T(H) .
$$

Так как $2^{t-1}+1$ делит $T\left(g_{1}(x) \ldots g_{l}(x)\right)$, верна цепочка равенств

$$
\begin{aligned}
2^{t-1}+1 & =\left(2^{t-1}+1, T\left(g_{1}(x) \ldots g_{l}(x)\right)\right) \\
& =\left(2^{t-1}+1,\left[T\left(g_{1}(x)\right), \ldots, T\left(g_{1}(x)\right)\right]\right) \\
& =\left[\left(2^{t-1}+1, T\left(g_{1}(x)\right)\right), \ldots,\left(2^{t-1}+1, T\left(g_{1}(x)\right)\right)\right] .
\end{aligned}
$$

Так как $g_{1}(x), \ldots, g_{l}(x)$ - неприводимые многочлены степеней соответственно $n_{1}, \ldots, n_{l}$, то $T\left(g_{i}(x)\right)$ делит $2^{n_{i}}-1$ для каждого $i=1, \ldots, l$. Теперь из предыдущих равенств следует, что $2^{t-1}+1$ делит число

$$
\left[\left(2^{t-1}+1,2^{n_{1}}-1\right), \ldots,\left(2^{t-1}+1,2^{n_{\iota}}-1\right)\right] .
$$

По следствию 4 число $\left(2^{t-1}+1,2^{n_{i}}-1\right)$ равно либо 1 , либо $2^{m_{i}}+1$, где $m_{i}$ делит $t-1$ и $(t-1) / m_{i}$ - нечетное число. Предположим, что каждый из полученных таким образом параметров $m_{i}$ меньше $t-1$. Тогда нетрудно видеть, что число (25), а значит, и число $2^{t-1}+1$, делит $\left[2^{(t-1) / p_{1}}+1, \ldots, 2^{(t-1) / p_{s}}+1\right]$, где $p_{1}, \ldots, p_{s}-$ все различные простые нечетные делители $t-1$. Это противоречит лемме 4. Таким образом, можно считать, что $m_{1}=t-1$, то есть

$$
\left(2^{t-1}+1,2^{n_{1}}-1\right)=2^{t-1}+1 .
$$

В таком случае по следствию $42(t-1)$ делит $n_{1}$. Так как степень $m_{f}(x)$ не превосходит $3 t$, очевидно, что $n_{1}$ строго меньше $3 t$. Отсюда ввиду условия $t>4$ следует, что $n_{1}=2(t-1)$.

Далее полагаем, что

$$
\operatorname{deg} g_{1}(x)=2(t-1)
$$

и значит,

$$
d_{1}=1
$$

На множестве последовательностей $\mathscr{R}_{\Omega}(f)$ определим отображение

$$
\delta: \mathscr{R}_{\Omega}(f) \rightarrow \mathscr{R}_{\Omega}(f)
$$


по правилу

$$
\delta(u)=v,
$$

где

$$
v(i)=u(i+1)
$$

Нетрудно проверить, что $\delta$ - линейное преобразование $\mathbf{Z}_{2}$-пространства $\mathscr{R}_{\Omega}(f)$, характеристический многочлен $\chi_{\delta}(x)$ и минимальный многочлен $m_{\delta}(x)$ которого совпадают с характеристическим и минимальным многочленами матрицы $S(f)$ соответственно. Известно, что если $g(x)$ - неприводимый делитель минимального многочлена $m_{\delta}(x)$ линейного преобразования $\delta$, то его степень делит размерность $\operatorname{dim} \operatorname{Ker} g(\delta)$ ядра преобразования $g(\delta)$. В частности, отсюда следует, что степень многочлена $g_{1}(x)$, равная $2(t-1)$, делит $\operatorname{dim} \operatorname{Ker} g_{1}(\delta)$, и так как $\operatorname{dim} \operatorname{Ker} g_{1}(\delta) \leqslant$ $\operatorname{dim} \mathscr{R}_{\Omega}(f)=3 t$, справедливо равенство

$$
\operatorname{dim} \operatorname{Ker} g_{1}(\delta)=2(t-1) .
$$

Из общих свойств линейных преобразований $[4,5]$ следует равенство

$$
\mathscr{R}_{\Omega}(f)=\operatorname{Ker} g_{1}(\delta)^{d_{1}} \dot{+} \ldots \dot{+} \operatorname{Ker} g_{l}(\delta)^{d_{l}} \text {. }
$$

Отсюда, ввиду леммы 7 и равенства (3.7), следует, что

$$
\begin{gathered}
\mathscr{R}_{\Omega}(f)=\operatorname{Ker} g_{1}(\delta) \dot{+} \operatorname{Ker}\left(g_{2}(\delta) \ldots g_{l}(\delta)\right)^{2}, \\
\operatorname{dim} \operatorname{Ker}\left(g_{2}(\delta) \ldots g_{l}(\delta)\right)^{2}=t+2 .
\end{gathered}
$$

Оценим мощность множества $\operatorname{Ker} g_{1}(\delta) \ldots g_{l}(\delta)$. Для этого выведем сначала некоторые соотношения. Для любого многочлена $g(x) \in \mathbf{Z}_{2}[x]$ соответствующее ему линейное перобразование $g(\delta)$ обладает очевидно следующими свойствами:

$$
\begin{aligned}
\operatorname{Ker} g(\delta) & \leqslant \operatorname{Ker} g(\delta)^{2}, \\
g(\delta) \operatorname{Ker} g(\delta)^{2} & \leqslant \operatorname{Ker} g(\delta) .
\end{aligned}
$$

По теореме об эпиморфизме групп

$$
\operatorname{Ker} g(\delta)^{2} / \operatorname{Ker} g(\delta) \cong g(\delta) \operatorname{Ker} g(\delta)^{2} .
$$

Следовательно,

$$
\begin{aligned}
\left|\operatorname{Ker} g(\delta)^{2} / \operatorname{Ker} g(\delta)\right| & =\frac{\left|\operatorname{Ker} g(\delta)^{2}\right|}{|\operatorname{Ker} g(\delta)|} \\
& =\left|g(\delta) \operatorname{Ker} g(\delta)^{2}\right| \\
& \leqslant|\operatorname{Ker} g(\delta)|
\end{aligned}
$$

и справедливо равенство

$$
\left|\operatorname{Ker} g(\delta)^{2}\right| \leqslant|\operatorname{Ker} g(\delta)|^{2}
$$


Отсюда и из (27), в частности, следует неравенство

$$
\operatorname{dim} \operatorname{Ker} g_{2}(\delta) \ldots g_{l}(\delta) \geqslant(t+2) / 2,
$$

и потому

$$
\begin{aligned}
\left|\operatorname{Ker} g_{1}(\delta) \ldots g_{l}(\delta)\right| & =\left|\operatorname{Ker} g_{1}(\delta)\right|\left|\operatorname{Ker} g_{2}(\delta) \ldots g_{l}(\delta)\right| \\
& \geqslant 2^{(5 t-2) / 2}>2^{2 t}
\end{aligned}
$$

Отсюда следует, что множество $\operatorname{Ker} g_{1}(\delta) \ldots g_{l}(\delta)$ содержит две различные последовательности, которые совпадают по первым двум членам. А это означает, что их сумма - некоторая ненулевая последовательность $u \in \operatorname{Ker} g_{1}(\delta) \ldots g_{l}(\delta)$ такая, что $u(0)=u(1)=0$. Как уже отмечалось ранее,

$$
T\left(g_{1}(x) \ldots g_{l}(x)\right)=\left(2^{t-1}+1\right) T(H)=\tau .
$$

Так как $u \in \operatorname{Ker} g_{1}(\delta) \ldots g_{l}(\delta)$, то $T(u)$ делит $\tau$, то есть

$$
u(\tau))=u(0)=0 .
$$

По следствию $5 T(H)$ - нечетное число, значит,

$$
\tau \equiv 2^{t-1}+1 \quad(\bmod q+2)
$$

и ввиду леммы 8 из условия

$$
u(\tau)=0
$$

следует, что

$$
u\left(2^{t-1}+1\right)=0
$$

Таким образом, в ненулевом слове

$$
(u(0), u(1), \ldots, u(q+1)) \in \mathscr{K}
$$

есть по крайней мере 3 нуля, то есть

$$
d(\mathscr{K}) \leqslant q-1
$$

Следовательно, линейного рекурсивного $[q+2,3, q]$-кода не существует. Это завершает доказательство теоремы 2.

Докажем следствие 2. Линейный в широком смысле код $\mathscr{X}$, эквивалентный расширенному $\left[2^{t}+2,3,2^{t}\right]$-коду Рида-Соломона, является МДР-кодом. Если он при этом циклический, то $\mathscr{K}$ - линейный 3-рекурсивный МДР-код, что противоречит теореме 2. Отсутствие кода, линейно в широком смысле эквивалентного $\left[2^{t}+2,2^{t}-1,4\right]$-коду, доказывается исходя из двойственности последнего к рассмотренному выше расширенному коду Рида-Соломона размерности 3.

Автор искренне признателен А. А. Нечаеву за постановку задачи, научное руководство работой и помощь в подготовке рукописи. 


\section{Список литературы}

1. Мак Вильямс Ф. Дж., Слоэн Н. Дж. А., Теория кодов, исправляющих ошибки. Связь, Москва, 1979.

2. Heise W., Quattrocci P., Informations und Codierings Theorie. Springer, Berlin, 1995.

3. Лиддл Р., Нидеррайтер Г., Конечные поля. Мир, Москва, 1988.

4. Гонсалес С., Коусело Е., Марков В. Т., Нечаев А. А., Рекурсивные МДР-коды и рекурсивно дифференцируемые квазигруппы. Дискретнал математика (1998) 10, №2, 3-29.

5. Couselo E., Gonzalez S., Markov V., and Nechaev A., Recursive MDS-codes. In: Proc. WCC'99. Paris, 1999, pp. 271-278.

6. Bush K. A., Ortogonal arrays of index unity. Ann. Math. Statist. (1952) 23, 426-434.

7. Нечаев А. А., Кузьмин А. С., Марков В. Т., Линейные коды над конечными полями и модулями. Фундаментальная и прикладная математика (1996) 3, №1, 195-254.

8. Kuzmin A. S., Kurakin V. L., Mikhalev A. V., Nechaev A. A., Linear recurrences over rings and modules. J. Math. Sci., Contemporary Math. Appl., Thematic Surveys. 1994, v.10.

Статья поступила 12.10.1999. 